\section{SHORT COURSES}

ASMS will sponsor five short courses on May 18 and 19,1991 , immediately preceding the annual conference in Nashville.

Introduction to the Interpretation of Mass Spectra, organized by the Washington, DC, Mass Spectrometry Discussion Group

Advanced Interpretation of Mass Spectra, organized by Fred McLafferty and Frank Turecek

LC/MS, organized by Al Yergey and John Coutant

Fundamentals of Protein Analysis, organized by Barbara Larsen and Charles McEwen

GC/MS Methods in Drug Testing, organized by Rodger Foltz, Jack Henion, and John Cody

\section{9th ASMS CONFERENCE}

You will find more than country music in Nashville, Tennessee, the site of the 39th ASMS Conference, May 19-24, 1991. The conference will be located in the magnificent Opryland Hotel, one of the largest convention hotels in the United States. Complete conference information may be obtained from the Society Office.

\section{ASMS CALENDAR}

March Conference preliminary program

April 26 Deadline for advance conference registration and short course registration

May 18-19

ASMS short courses

May 19-24 39th ASMS conference

\section{THE ASILOMAR CONFERENCE}

September 23-27, 1990, Report prepared by Veronica M. Bierbaum

The Asilomar Conference on Mass Spectrometry (ACMS), an affiliate of ASMS, provides a forum for the presentation and discussion of the most recent advances in a subfield of mass spectrometry with the intent of stimulating new ideas. It complements the broader, open-attendance Annual Meeting on Mass Spectrometry and Allied Topics.

The 8th ACMS was held September 23-27, 1990, on the topic of "Intermediates in Gas Phase Ion Chemistry." The conference proved to be superb in every way; the scientific program was exceptional and the organizational aspects were excellent.

Helmut Schwarz, Program Chairman, gathered an international group of distinguished mass spectrometrists, spectroscopists, and theoreticians who discussed new developments in the field of ion chemistry. The program consisted of thirty lectures, including a plenary lecture by Nobel Laureate Professor $Y$. T. Lee, and nineteen posters. Topics included the reaction kinetics, mechanisms, thermodynamics, structures, and transport properties of a wide variety of ionic species including distonic ions, ion-dipole complexes, metal ions, cluster ions, and organic negative ions. Lectures were presented by P. B. Armentrout, T. Baer, J. L. Beauchamp, D. K. Bohme, K. H. Bowen, M. T. Bowers, J. I. Brauman, C. H. DePuy, K. Eller, M. A. ElSayed, K. M. Ervin, B. S. Freiser, M. L. Gross, H.-Fr. Grützmacher, S. Hammerum, J. F. Harrison, S. R. Leone, C. Lifshitz, J. C. Lorquet, F. W. McLafferty, J. Michl, N. M. M. Nibbering, R. O'Hair, L. Radom, H. Schwarz, R. R. Squires, J. K. Terlouw, J. C. Weisshaar, and $F$. Williams.

The pleasant and informal atmosphere of the Asilomar Conference Center encouraged lively and productive interactions among the scientists. The 118 attendees expressed appreciation to Laslo Tökés, Chairman, and to the other members of the ACMS Board of Directors for their efforts in developing this exciting and valuable conference.

\section{THE ASMS FALL WORKSHOP}

November 5-6, 1990, Report prepared by Ian Jardine

Electrospray ionization has revolutionized the ability of mass spectrometrists to analyze large biological molecules at high sensitivity. In addition, the ionization technique is clearly a major advance in the coupling of separations systems such as high performance liquid chromatography (HPLC) and high performance capillary electrophoresis (HPCE) to mass spectrometry.

To paraphrase Bob Murphy and the ASMS Board, the idea of this particular workshop was to provide information on how one could build an electrospray source, get it to operate, and provide insight into how to couple electrospray with various separation techniques. The general idea of the workshop is to provide a forum for interchange between attendees and experts in the field. 


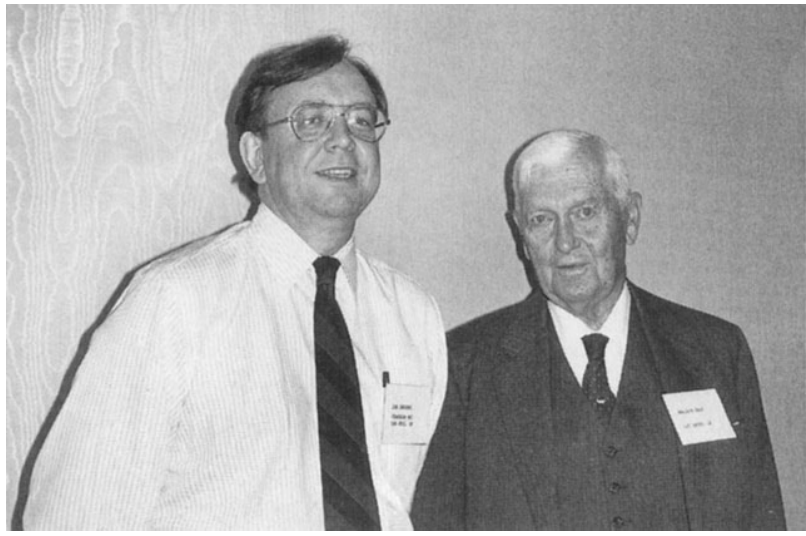

Ian Jardine and Malcolm Dole at the Fall Workshop on Electrospray. We are saddened to announce the death of Dr. Dole in December.

With this caveat in mind, the workshop began with the introduction of Malcolm Dole to the audience. Malcolm's prescient work in the 1960s laid the groundwork for today's electrospray techniques. Sadly, Malcolm Dole died peacefully at the age of 87 at his home in Los Gatos, California, in early December

Mark Allen of Vestec Corporation then launched into the history of the development of electrospray ionization, various ways that electrospray had been developed, and the progress on electrospray ionization coupled to quadrupole, magnetic sector, Fourier transform, and quadrupole ion trap systems. Mark detailed his own experiences of coupling a unique electrospray system to a quadrupole instrument. Brian Chait of the Rockefeller University described the unique electrospray system developed at Rockefeller for quadrupole analyzers. To keep the advantages and limitations of electruspray ionization in perspeclive, Brian compared the electrospray ionization technique with matrix-assisted time-of-flight mass spectrometry, which is also being used extensively in his lab, often on the same protein samples. Of particular interest was the ability of laser desorption-time-of-flight mass spectrometry to analyze large and very heterogeneous glycoproteins, even directly from salts and other matrices at total analyte amounts of 100 fmole - 1 pmole, a task that electrospray currently cannot match. Brian also provided many practical hints on how to obtain successfully electrospray ionization spectra for real protein samples.

The evening poster session displayed twelve posters illustrating various developments such as electrospray ionization on magnetic sector systems, characterization of glycosylated proteins by liquid chromatography combined with mass spectrometry (LC/MS), applications of online HPCE/electrospray ionization and HPLC/electrospray ionization, quantitation of proteins in serum, electrospray on an ion trap, detailed carbohydrate structural analysis by using advanced software treatment on electrospray ionization data, characterization of glucuronide and glutathione conjugates, and analysis of organometallic complexes.
The next day's session began with Tom Covey of Sciex describing the electrospray/ion spray system in detail and its use for LC/MS using various HPLC column systems. Tom discussed many impressive examples of the application of this system for peptide, glycopeptide, drug, and other organic molecules by LC/MS and LC/MS/MS analysis. Tom also discussed the application of LC/MS with electrospray ionization for quantitation of drugs from biological matrices. The technique is sensitive and will clearly be extremely useful for quantitative analysis, especially by using appropriate internal standards, even though the system has limited dynamic range of $\sim 3$ orders of magnitude or so. Paul Kebarle of the University of Alberta then discussed the mechanisms of desorption using clear and relevant experimental data from his own lab.

Jeff Shabanowitz of the University of Virginia described his experiences in coupling micro-HPLC as well as HPCE with a quadrupole MS/MS system and explained sample handling and other practical issues involved to allow the online analysis of peptides at the low picomole to femtomole level.

Michael Geisow, a biotechnologist, gave his perspective on why he thought electrospray ionization was a revolutionary technique for his discipline. In particu lar, by using some nice examples of the analysis of proteins generated in large scale in a production facility, he explained how the mass accuracy and ability to analyze protein mixtures to determine anomalies in protein production would be invaluable for his field.

Ian Jardine concluded with a discussion of some issues not covered by the previous speakers, such as other ways of coupling of LC to the electrospray ionization combined with mass spectrometry system for difficult solvents such as $100 \%$ water; software advances for the analysis of mass spectrometry, MS/ MS, and LC/MS data; salient issues regarding protein heterogeneity and the need for higher mass resolution analysis; and, by using data from Battelle Northwest Labs, the current state of the art for electrospray ionization analysis of RNA and DNA.

There was much room for discussion and, indeed, many other important issues arose in these sessions. For example, it is clear that mass spectrometrists need to be careful in some applications of electrospray, such as when analyzing peptides and proteins. The user is creating an aerosol of these potentially toxic and immunogenic materials, so there is a clear danger of exposure. The user, therefore, should be protected from the spray. In addition, some users may experiment with spraying diverse solvents of a potentially toxic or hazardous nature and, again, caution and appropriate safety measures are mandatory.

The ASMS News is prepared by Judith A. Watson, Executive Director. For additional information contact ASMS, P.O. Box 1508, East Lansing, MI 48826. Telephone: 517-337-2548. 